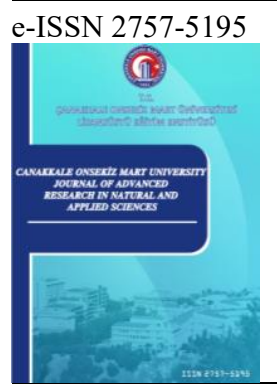

Çanakkale Onsekiz Mart University

Journal of Advanced Research in Natural and Applied Sciences

Open Access

\title{
Kavak Çayı (Gelibolu, Çanakkale ) Mollusca Faunası ve Bazı Su Kalitesi Özelliklerinin Belirlenmesi
}

\author{
Demet Bal ${ }^{1}$, Deniz Anıl Odabaş1²*, Aytuğ Zilifli ${ }^{3}$ \\ ${ }^{1}$ Sınırlı Sorumlu Su Ürünleri Kooperatifleri Birliği, No:2, Efeler, Aydın, Türkiye \\ ${ }^{2}$ Deniz Bilimleri ve Teknolojisi Fakültesi, Çanakkale Onsekiz Mart Üniversitesi, Çanakkale, Türkiye \\ ${ }^{3}$ Lisansüstü Eğitim Enstitüsü Çanakkale Onsekiz Mart Üniversitesi, Çanakkale, Türkiye \\ $\begin{array}{lr}\text { Makale Tarihçesi } \\ \text { Gönderim: } & 09.02 .2021 \\ \text { Kabul: } & 19.08 .2021 \\ \text { Yayım: } & 20.09 .2021\end{array}$ \\ Araștırma Makalesi \\ $\ddot{\mathbf{O z}}$ - Marmara havzasının Batısında bulunan Kavak Çayının sucul Gastropoda ve Bivalvia faunası ile bazı su kalitesi özellik- \\ lerinin araştırılmasını amaçlayan bu çalışmada, Kavak Çayı havzasından seçilen beş istasyondan 2016 yılı içerisinde, makro- \\ omurgasız taban faunası ve su örneklemeleri mevsimsel olarak gerçekleştirilmiştir. Standart örnekleme yöntemleri kullanılarak \\ yapılan taban faunası örneklemelerinden sonra taban faunasındaki gastropod ve bivalv örneklerine ait bireylerin tür teşhis ve \\ sayımları laboratuvarda gerçekleștirilmiștir. Molluska türlerinin istasyonlara göre birey sayısı, baskınlık ve frekans değerleri \\ hesaplanmıştır. Bunun yanında, tespit edilen türlerin tolerans değerleri de verilmiştir. Örnekleme istasyonlarında yerinde öl- \\ çülen su sıcaklığı, çözünmüş oksijen (doygunluk ve yoğunluk), pH, toplam çözünmüş katılar, elektrik iletkenliği gibi paramet- \\ reler kaydedilmiştir. Diğer su kalitesi parametrelerinden nütrient ve bazı elementler ise laboratuvarda iyon kromotografisi \\ cihazı ile analiz edilmiştir. Çalışma sonuçlarına göre, araştırma bölgesinde sucul molluska faunasına ait toplam 17 takson \\ belirlenmiștir. Yerinde ve laboratuvarda ölçülen su kalitesi parametreleri Türkiye Su Kalitesi Kontrolü Yönetmenliğine göre \\ karşılaştırıldığında, örnekleme istasyonlarının ölçülen parametrelere göre birinci (en yüksek) ve dördüncü kalite (en düşük) \\ sınıfları arasında oldukları belirlenmiştir. Özellikle çözz̈nmüş oksijen ve Alüminyum gibi bazı parametrelerin etkisiyle su \\ kalitesi sınıflarının düștüğü gözlenmiștir. Sonuç olarak, Kavak Çayı ve havzasında belirli su kalite parametrelerinin su kalite- \\ sini kötüleştirdiği, buna bağlı olarak da, ortamda yaşayan molluska faunası kompozisyonunun genel olarak organik kirliliğe \\ toleranslı türlerden oluştukları görülmüştür.
}

\section{Determination of Mollusca Fauna and Some of The Water Quality Featu- res of Kavak Stream (Gelibolu Peninsula: Çanakkale)}

${ }^{1}$ Limited Liability of Fisheries Cooperatives Association, No:2, Efeler District, Aydın, Turkey $2^{2 *}$ Faculty of Marine Science and Technology, Çanakkale Onsekiz Mart University, Çanakkale, Turkey ${ }^{3}$ School of Graduate Studies , Çanakkale Onsekiz Mart University, Çanakkale, Turkey

\author{
Article History \\ Received: $\quad 09.02 .2021$ \\ Accepted: $\quad 19.08 .2021$ \\ Published: $\quad 20.09 .2021$
}

Research Article

\begin{abstract}
This study aimed at determining the Mollusca fauna and some water quality features of Kavak Stream located on the Western part of the Marmara Basin. Therefore, sampling studies on macrozoobenthos and water were conducted on the pre-selected five sampling stations seasonally, in the year of 2016. After the benthos samplings carried out using standard methods, taxonomic identifications of the gastropods and bivalves were done and individual numbers were carried out in the laboratory. Abundance, dominance, and frequency of the Mollusca were calculated. Besides, taxa tolerance values of the identified taxa were also given. Some physical and chemical parameters of water such as temperature, oxygen saturation, $\mathrm{pH}$, dissolved solids, and conductance were recorded during the field study. Other water quality parameters such as nutrients and some elements were analysed in the laboratory using ion chromatography. According to the data of the present study, seventeen taxa were identified belong to the aquatic Mollusca fauna. The environmental variables both recorded in the field and analysed in the laboratory were between first (the highest) and fourth (the lowest) class when compared with Water Quality Criteria Legislation of Turkey. Especially, the dissolved oxygen and Aluminium concentrations decreased the water quality class in the stations was observed. In conclusion, deteriorated the water quality under the influence of some parameters, accordingly the inhabitant Mollusca fauna composition were tolerant to organic pollution was observed.
\end{abstract}

\footnotetext{
1 (iD) sahra092@hotmail.com

(i) aodabasi@ comu.edu.tr

3 (D) aytugziliflii@gmail.com

*Sorumlu Yazar / Corresponding Author
} 


\section{Giriș}

Sucul ekosistemler sürekli olarak çeşitli kaynaklardan gelen kirletici insan faaliyetlerinin etkisi altındadır (Salanki, Salanki, Kamardina ve Rozsa, 2003). Sucul ekosistemler içinde akarsular ise en fazla baskısıya maruz kalan ve ilk olarak etkilenen ortamlardır. Bu nedenle sürekli olarak su kimyası kalitesi ve ekolojik kalite anlaminda kontrol edilmesi gerekmektedir ( Hering vd., 2006). Biyolojik indikatörler, sucul ekosistemlerin kalitesinin izlenmesinde uzunca bir süredir kullanılmaktadır (Rosenberg ve Resh, 1993). Su zeminine bağlı olarak yaşayan (taban omurgasızları, fitobentoz, makroalg gibi) komünitlerin çevresel değişkenlerle ile etkileşimleri, yalnızca mevcut durumu değil aynı zamanda uzun bir zaman dilimi içerisinde oluşan etkilerin gösterilmesini de sağlamaktadır (Rosenberg, 1998).

Sucul mollusklar, taban omurgasızları içerisinde ekolojik ve biyolojik izleme çalışmalarında, hemen hemen tüm sucul ortamlarda bulunabilmeleri, yüksek çeşitliliğe sahip olmaları ve kirleticilere karşı az ya da çok tepki göstermesi ile öne çıkan bir canlı grubudur (Goldberg, 1986; Salanki, 1989). Bazı taksonomik gruplar (familya seviyesinde) sınırlı tolerans gösterse de, bazıları geniş toleransa sahiptir (Yıldırım, 1999; Mandaville, 2002). $\mathrm{Bu}$ nedenle sıklıkla pasif ve aktif biyolojik izleme ve risk değerlendirme çalışmalarında kullanılmaktadır (Borcherding ve Volpers, 1994).

Türkiye gibi gelişmekte olan ülkelerde doğrudan veya arıtılarak sucul ortamlara salınan evsel ve endüstriyel atıklar giderek artmakta olan bir problemdir (Demirbaș, 2001). Buna bağlı olarak, endüstrileşme ve hızlı nüfus artış1, özellikle son y1llarda önemli bir sorun haline gelmiştir (Karslığlu, Baba ve Ozan, 2004). Ülkemiz su kaynaklarının korunması ve iyi ekolojik duruma getirilmesi çalışmaları, Avrupa Birliği’ne uyum programı ile hız kazanmış, bu bağlamda Su Çerçeve Direktifi (SÇD) (2000/60/AT) ülkemizde uygulamakta ve yüzey sularının fiziko-kimyasal ve biyolojik izleme programları yürütülmektedir (Bulut ve Birben, 2019). Biyolojik izleme programlarında en çok kullanılan ve SÇD kapsamında bir kalite unsuru olan taban büyük omurgasızları su kütlesi hakkında kapsamlı bir değerlendirme imkânı sunmaktadır (Karr, 1999; Kenney, Sutton-Grier, Smith, ve Gresens, 2009; Kazanc1 vd., 2010). Taban omurgasızlanı içinde önemli bir yeri olan tatlısu molluskları hakkında ülkemizde yapılan ilk çalışmalar Roth (1839) ile başlamış, müteakiben yerli ve yabancı çok sayıda bilimsel çalışmayla (Schütt, 1964; Schütt ve Bilgin, 1970; Bilgin, 1980; Schütt ve Șeșen, 1993; Yıldırım, 1999; Ustaoğlu, Balık ve Özbek, 2001, 2003; Öktener, 2004; Y1ldırım vd., 2006; Kebapçı ve Y1ldırım, 2010; Kıl1çaslan ve Özbek, 2010; Koșal-Șahin, Bahadır-Koca ve Yıldırım, 2012; Odabaș1 ve Georgiev, 2014; Yıldırım. Kebapçı, Koca ve Yüce, 2015; Koşal-Şahin ve Zeybek, 2016; Gürlek, 2017; Gürlek, Koşal-Șahin, Dökümcü ve Yıldırım, 2019; Gözler ve Baytașoğlu, 2020; Tomilova vd., 2020; Odabaș1, Akay ve Koyuncuoğlu, 2020; Sands vd., 2020) hız kazanmıştır.

Marmara bölgesinin önemli bir bölümünü temsil eden Çanakkale İlinin kapladığı alandaki sucul ekosistemlerde oldukça sınırlı sayıda ekolojik çalışma mevcuttur. Diğer taraftan Gelibolu Yarımadası (Çanakkale) sınırlı Tatlısu kaynaklarının varlığı ile dikkat çeken bölgelerden birisidir. Biga Yarımadası içsu havzalarında bazı faunistik (Bilgin, 1980; Sarı, Balık, Ustaoğlu ve Ilhan, 2006; Odabaşı, Kebapçı ve Akbulut, 2013; Odabaşı ve Georgiev, 2014; Odabaș1, Arslan, Özdilek ve Odabaș1, 2019) ve ekolojik (Akbulut vd., 2009a; Akbulut vd., $\underline{2009 b}$; Odabașı ve Büyükateș, 2009) konuları ele alan bilimsel çalışmaların varlığı görülse de, Çanakkale'nin Avrupa kısmındaki en önemli doğal sucul ekosistemi olan Kavak Çayı'nda çok az çalışma mevcut olup (Kelkit ve Öztürk, 2005; Akbulut, Odabaşı, Odabaşı ve Çelik, 2006), sucul omurgasızları hakkında hiç bilimsel veri bulunmamaktadir.

Kavak Çayı ve havzasının bazı kollarında gerçekleştirilen bu çalışmada, sucul molluska tür çeşitliliği ile bazı su kalitesi parametrelerinin belirlenmesi amaçlanmıştır. Çalışma alanını oluşturan bölgede bu konu ile ilgili bir bilimsel çalışma bulunmamasından dolayı ilk olma özelliği taşıyan bu araştırma, bölgenin ve ülkemizin zoocoğrafik bilgi birikimine katkı sunabilecek niteliktedir. 


\section{Materyal ve Yöntem}

\section{1. Örnekleme Bölgesi}

Marmara bölgesinin Trakya bölümünde ve Çanakkale ili ile Tekirdağ ili arasında yer alan Kavak Çayı, çalışma alanını oluşturmaktadır. $50 \mathrm{~km}$ uzunluğundaki Kavak Çayı Gelibolu Yarımadasını geçip Saroz körfezi' ne dökülür. Korudağı' ndan doğan Kavak Çayı, $1100 \mathrm{~m}^{3} / \mathrm{sn}$ 'lik bir su debisine sahiptir (Anonim, 2013). Havzayı etkileyen başlıca kirleticilerin evsel atıklar, evsel katı atıklar, zirai ilaç ve gübre kullanımlarının olduğu belirtilmektedir. Kavak Çayı Deltası, 2010 yılında Özel Çevre Koruma Alanı olarak kabul edilmiştir, bunun yanı sıra birinci derece doğal sit alanıdır. Ülkemizdeki önemli 135 sulak alan içinde kalmaktadır (Anonim, 2013).

Bu çalışmanın amacına yönelik olarak, Kavak Çayı havzasında 5 farklı örnekleme istasyonu seçilmiştir. Kavak deltasına yakın bir konumda bulunan birinci örnekleme istasyonu Kavakköy yakınından belirlenmiştir. Birinci istasyona yakın konumdaki Kavakköy köprüsü, ikinci örnekleme istasyonu seçilmiştir. Evreşe Beldesinin yakınındaki alandan seçilen bölge, çalışmanın üçüncü örnekleme istasyonudur. Dördüncü örnekleme istasyonu, Bayramiç Beldesi yakınından belirlenmiştir. Yülüce Köyü yakınından belirlenen örnekleme alanı ise bu çalışmanın beşinci ve son istasyonudur (Şekil 1, Tablo 1).

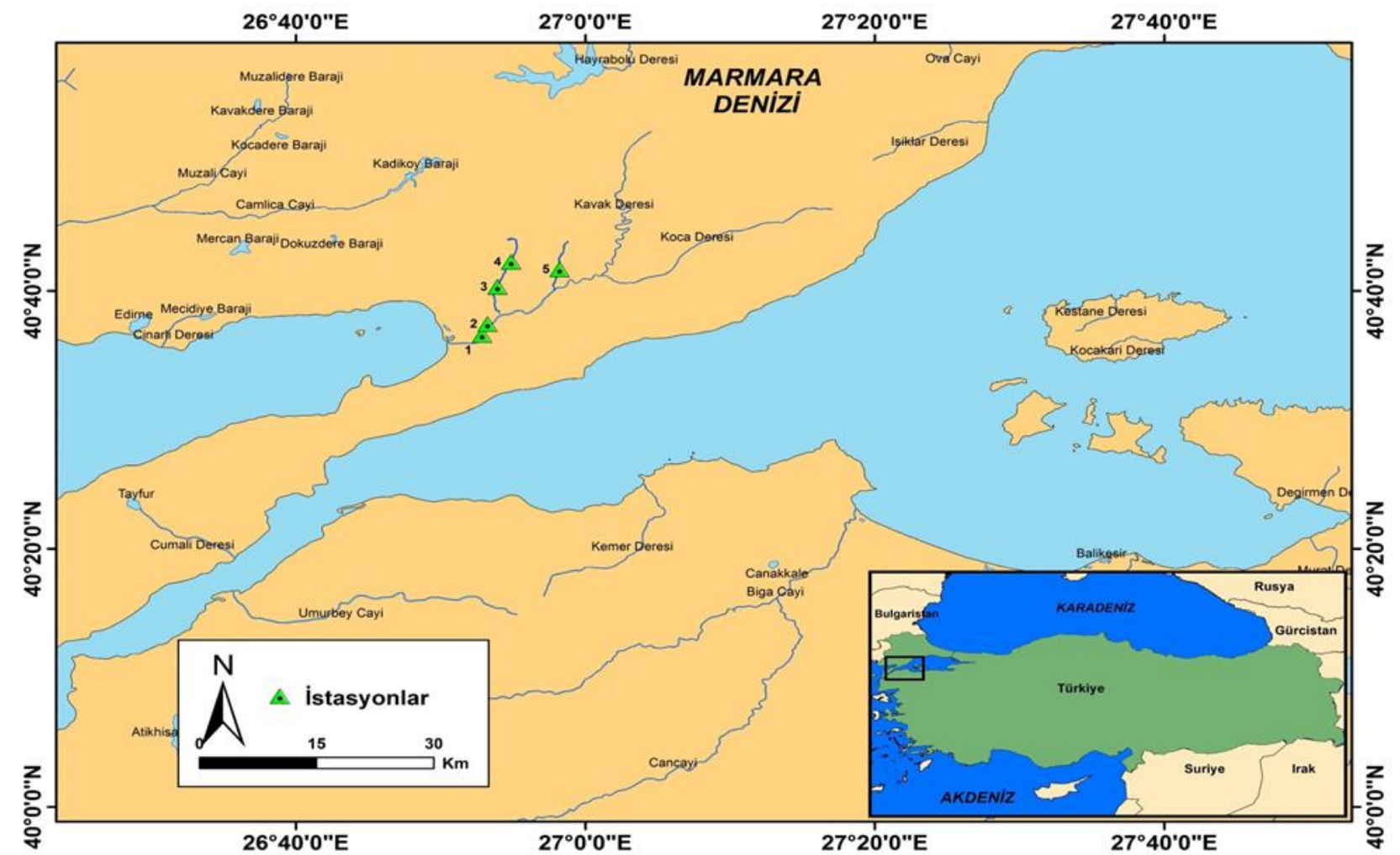

Şekil 1. Çalışma bölgesinde seçilen örnekleme noktaları 
Tablo 1

Çalışma alanında seçilen istasyonların koordinatları ve örnekleme tarihleri

\begin{tabular}{|c|c|c|}
\hline İstasyonlar & Örnekleme zamanları & Koordinat \\
\hline KV1 & $\begin{array}{l}\text { 29.01.2016, 24.05.2016, } \\
31.08 .2016,03.11 .2016\end{array}$ & $40^{\circ} 37^{\prime} 23^{\prime \prime} \mathrm{N} / 26^{\circ} 53^{\prime} 9^{\prime \prime} \mathrm{E}$ \\
\hline KV2* & $24.05 .2016,03.11 .2016$ & $40^{\circ} 36^{\prime} 49^{\prime \prime} \mathrm{N} / 26^{\circ} 53^{\prime} 0^{\prime \prime} \mathrm{E}$ \\
\hline KV3* & 29.01.2016, 24.05.2016 & $40^{\circ} 40^{\prime} 12^{\prime \prime} \mathrm{N} / 26^{\circ} 53^{\prime} 32^{\prime \prime} \mathrm{E}$ \\
\hline KV4 & $\begin{array}{l}\text { 29.01.2016, 24.05.2016, } \\
31.08 .2016,03.11 .2016\end{array}$ & $40^{\circ} 42^{\prime} 3^{\prime \prime} \mathrm{N} / 26^{\circ} 54^{\prime} 17^{\prime \prime} \mathrm{E}$ \\
\hline $\mathbf{K V 5}^{* *}$ & $29.01 .2016,24.05 .2016$ & $4041^{\prime} 39^{\prime \prime}$ N/ $26^{\circ} 58^{\prime} 9^{\prime \prime} \mathrm{E}$ \\
\hline
\end{tabular}

*Akarsu yatağı çevresindeki alt yapı çalışmaları nedeniyle her dönem örnekleme yapılmamıştır

**Örnekleme noktasında su akışı olmadığı dönemlerde örnekleme yapılmamıştır

\subsection{Bentos Örneklemesi ve Tür Teşhisleri}

Molluska tür çeşitliliğinin belirlenmesi amacıyla akarsu tabanı çoklu habitat yöntemi (BS EN 16150:2012) ile örneklenmiştir. Yöntem, her bir akarsu içi habitatın (Kumlu, Çamurlu, Bitkili, Detritus) akarsu boyunca 20 m mesafe içindeki kapladığı alanın büyüklüğüne göre el kepçesi $(25 \mathrm{~cm}$ taban uzunluğu ve $500 \mu$ ağ göz açıklığı) ile örneklenmiştir. Bununla birlikte örnekleme alanında bulunan taş ve bitkilik habitatlar incelenmiş, bulunan farklı türdeki örnekler pens, firça gibi aletler yardımıyla örneklere dahil edilmiştir. Örneklerin fiksasyon için \%80'lik etil alkol kullanılmıştır. Laboratuvara getirilen bentik örnekler organizma gruplarına ayrılmıştır. Gruplara ayrılan organizmalar Stereo zoom (Olympus SD30) mikroskop kullanılarak teşhis edilmiştir. Teşhisler Glöer (2015), Killeen, Aldridge, Oliver ve Council (2004), Bilgin (1980), Kornuishin (1996), Schütt (1965), Öztürk, Önen ve Doğan (2008) ve Lopes-Lima vd. (2021)'e göre yapılmıştır. Türlerin fotoğraflanması ve ölçeklendirilmesinde Zeiss Stemi 508 ile bütünleşik Axiocam 105 color cihazı kullanılmıştır.

\subsection{Suyun Fiziksel ve Kimyasal Değişkenleri}

Bentik örnekleme yapılan örnekleme istasyonlarında suyun $\mathrm{pH}$, çözünmüş oksijen, TDS (toplam çözünmüş katılar) parametreleri çoklu parametre ölçer (HI 98194, pH/EC/DO multiparameter) ile yerinde kaydedilmiştir. Ayrıca $500 \mathrm{~mL}$ 'lik polietilen örnekleme kaplarına alınan su örnekleri de $\mathrm{Mg}^{+2}, \mathrm{Ca}^{+2}, \mathrm{Cl}^{-}, \mathrm{NH}_{4}{ }^{+}, \mathrm{N}, \mathrm{NO}_{3}{ }^{-}, \mathrm{PO}_{4}$, $\mathrm{B}, \mathrm{Ni}, \mathrm{Co}, \mathrm{Zn}, \mathrm{Fe}, \mathrm{Al}, \mathrm{Ba}, \mathrm{Mn}, \mathrm{Cr}, \mathrm{Cu}, \mathrm{Pb}, \mathrm{Cd}$ ve $\mathrm{Na}^{+} \mathrm{K}, \mathrm{Ca}$ iyonlarının analizleri için Çanakkale Onsekiz Mart Üniversitesi Merkez Laboratuvarına soğuk zincirde transfer edilmiştir. Su parametreleri Su Kalitesi Kriterleri yönetmeliği (Anonim, 2004) ile karşılaştırılıp değerlendirilmiştir.

\subsection{Veri Analizi}

Teşhis edilen tatlısu molluskları istasyonlardaki bulunuşlarına göre baskınlık ve sıklık değerleri PAST (4.05) paket programı ile hesaplanmıştır. Su kalitesi parametrelerinin istasyonlardaki değerlerinin mevsim ortalamaları ve standart sapma değerleri belirlenmiştir.

\section{Bulgular ve Tartışma}

Çalışma boyunca gerçekleştirilen örneklemelerde teşhis edilen toplam takson sayısı 17'dir. Gastropoda sinıfina ait Bithynia tentaculata, Bithynia sp., Ecrobia ventrosa, Bittium reticulatum, Valvata piscinalis ve Rissoa sp., Galba truncatula, Gyraulus piscinarum, Planorbis carinatus, P. intermixtus, Physella acuta, Radix labiata olmak üzere toplam 12 takson teşhis edilmiştir. Bivalvia sınıfından ise Pisidium nitidum, Musculium lacustre, Pisidium sp. ve Abra sp. ile Unio eucirrus olmak üzere 5 taksonun varlığı belirlenmiştir (Tablo 2). 
Tablo 2

Teşhis edilen sucul mollusklar ve bulundukları örnekleme istasyonları

\begin{tabular}{llllll} 
Türler / İstasyonlar & KV1 & KV2 & KV3 & KV4 & KV5 \\
\hline Gastropoda/ Caenogastropoda & & & & & \\
\hline Bithynia tentaculata & + & - & + & - & - \\
Bithynia sp. & + & - & - & - & - \\
Valvata piscinalis & + & - & - & - & - \\
Bittium reticulatum & + & - & + & - & - \\
Ecrobia ventrosa & + & - & - & - & - \\
Rissoa sp. & + & - & + & - & - \\
Gastropoda/Heterobranchia & & & & & \\
\hline Galba truncatula & + & + & + & - & - \\
Gyraulus piscinarum & + & - & - & - & - \\
Physella acuta & + & - & - & - & - \\
Planorbis carinatus & - & - & + & - & - \\
Planorbis intermixtus & + & - & - & - & - \\
Radix labiata & + & - & - & - & - \\
Bivalvia & & & & & \\
\hline Musculium lacustre & - & - & - & - & + \\
Pisidium nitidum & - & - & + & - & - \\
Pisidium sp. & + & - & + & - & - \\
Abra sp. & + & - & - & - & - \\
Unio eucirrus & + & - & - & - & - \\
\hline
\end{tabular}

Çalışma boyunca tespit edilen türlerden birey sayısı olarak en bol türlerin $\% 19.84$ baskınlık değeri ile Galba truncatula ile Valvata piscinalis olduğu belirlenmiştir (Tablo 3). Sayıca fazla olan bu gastropodlardan $V$. piscinalis' in İstasyon I ve İstasyon IV'ten örneklendiği belirlenmiştir. G. truncatula' nın ise sirasiyla en fazla istasyon IV ve istasyon III'ten örneklendiği görülmüştür (Tablo 3). Birey sayısı yüksek olan gastropodlar ile birlikte, V. piscinalis, Pisidium sp. ve Physella acuta \%22.22 frekans değeri ile çalışma boyunca en s1k rastlanılan taksonlardır (Tablo 3). Bivalvia grubuna ait taksonlardan sayıca en bol olanının \%19.05 dominans oranı ile Pisidium nitidum'un öne çıktığı görülmektedir. Bu türün en fazla birey sayısı İstasyon IV istasyonunda tespit edilmiştir. Akbulut vd. (2009a)'a göre Atikhisar Barajı ve Sarıçay (Çanakkale)'da yapılan çalışmada Molluska faunasına ait toplam 29 takson tespit edilmiştir. Atikhisar Barajı ve Sarıçay'da gerçekleştirilen bu çalışmada Physella acuta türünün \%11.76 baskınlık ve \%15.63 frekans değeri ile en baskın ve en sık rastlanan gastropod olduğu ortaya konulmuştur. Physella acuta Kavak Çayı'nda düşük birey sayısı ile temsil edilmesine rağmen frekansı en yüksek türdür. İki çalışma arasında tür çeşitliliği bakımından benzerlik bulunsa da, Sarıçay ve Atikhisar Barajında daha yüksek bir tür çeşitliliği görülmektedir. Bu iki çalışma, bivalv taksonları bakımından karşılaştırıldığında, Kavak Çayın'da Pisidium nitium türünün, Sarıçay akarsuyu ve Atikhisar Barajında ise Dreissena polymorpha türünün baskınlık ve sıklık bakımından belirgin biçimde öne çıktıkları görülmektedir. $P$. acuta ve D. polymorpha istilacı canlılar arasında gösterilen, sırasıyla Kuzey Amerika kıtası ve Doğu Avrupa kökenli mollusklardır (Dillon, Wethington, Rhett ve Smith, 2005; Stanczykowska, Lewandowski ve Ejsmont-Karabin, 1983). Çanakkale İlindeki (Anadolu tarafındaki) akarsularda varlığ tespit edilen D. polymorpha, Potamopyrgus antipodarum ve $P$. acuta gibi bazı istilac1 mollusk taksonlarının (Odabașı, Arslan, Özdilek ve Odabașı, 2019), sonuncusu hariç Kavak Çayı'nda var olmadığ görülmüştür. 
Tablo 3

İstasyonlarda tespit edilen canlı türlerinin mevsimlere göre dağılımı

MEVSIM

ÍLKBAHAR YAZ

SONBAHAR KIŞ

ISTASYON

$\begin{array}{llllllllll}1 & 2 & 3 & 4 & 5 & 1 & 2 & 3 & 4 & 5\end{array}$

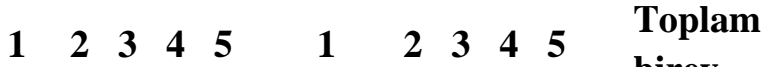

birey

$\% \mathrm{D} \quad \% \mathrm{~F}$

Gastropoda/ Pulmonata

Galba truncatula

$\begin{array}{lllll}0 & 0 & 0 & 27 & 0\end{array}$

$\begin{array}{lllll}4 & 0 & 0 & 10 & 0\end{array}$

$\begin{array}{lllll}1 & 0 & 0 & 0 & 0\end{array}$

$\begin{array}{llllll}0 & 0 & 8 & 0 & 0 & 50\end{array}$

$19.84 \quad 5$

27.78

Gyraulus piscinarum

$\begin{array}{llllllllll}0 & 0 & 0 & 0 & 0 & 2 & 0 & 0 & 0 & 0\end{array}$

$\begin{array}{lllll}0 & 0 & 0 & 0 & 0 \\ 3 & 1 & 0 & 0 & 0\end{array}$

$\begin{array}{lllll}0 & 0 & 0 & 0 & 2\end{array}$

0.79

5.56

Physella acuta

$\begin{array}{llllllllll}0 & 0 & 0 & 0 & 0 & 0 & 0 & 0 & 2 & 0\end{array}$

$\begin{array}{lllll}3 & 1 & 0 & 0 & 0\end{array}$

$\begin{array}{lllll}0 & 0 & 0 & 0 & 7\end{array}$

2.78

0.4

0.4

1.59

22.22

Planorbis intermixtu

$\begin{array}{llllllllll}0 & 0 & 0 & 0 & 0 & 0 & 0 & 0 & 0 & 0\end{array}$

$\begin{array}{llllll}1 & 0 & 0 & 0 & 0\end{array}$

$\begin{array}{llllll}0 & 0 & 0 & 0 & 0 & 1\end{array}$

Radix labiata

$\begin{array}{lllll}0 & 0 & 0 & 0 & 0\end{array}$

$\begin{array}{lllll}0 & 0 & 0 & 0 & 0\end{array}$

$\begin{array}{lllll}3 & 1 & 0 & 0 & 0\end{array}$

$\begin{array}{lllll}0 & 0 & 0 & 0 & 4\end{array}$

$\begin{array}{lllll}5 & 0 & 0 & 0 & 0\end{array}$

$\begin{array}{llllll}0 & 0 & 0 & 0 & 0 & 6\end{array}$

Bithynia tentaculata

$\begin{array}{lllll}0 & 0 & 0 & 1 & 0\end{array}$

$\begin{array}{lllll}0 & 0 & 0 & 0 & 0\end{array}$

$\begin{array}{lllll}5 & 0 & 0 & 0 & 0\end{array}$

Bithynia sp.

Valvata piscinalis

Bittium reticulatum

$\begin{array}{llllllllll}0 & 0 & 0 & 0 & 0 & 0 & 0 & 0 & 0 & 0\end{array}$

$\begin{array}{lllll}35 & 0 & 0 & 0 & 0\end{array}$

$\begin{array}{lllllllll}0 & 0 & 0 & 0 & 3 & 0 & 2 & 10 & 0\end{array}$

$\begin{array}{lllll}15 & 0 & 0 & 0 & 0\end{array}$

$\begin{array}{llllll}0 & 0 & 0 & 0 & 0 & 5\end{array}$

$\begin{array}{lllllllllll}0 & 0 & 0 & 8 & 0 & 0 & 0 & 0 & 0 & 0\end{array}$

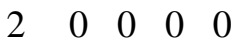

$\begin{array}{llllll}0 & 0 & 0 & 0 & 50\end{array}$

$\begin{array}{lllll}0 & 0 & 0 & 0 & 0\end{array}$

$\begin{array}{lllll}4 & 0 & 0 & 0 & 0\end{array}$

$\begin{array}{lllll}9 & 0 & 0 & 0 & 0\end{array}$

$\begin{array}{llllll}4 & 0 & 0 & 0 & 0 & 37\end{array}$

Rissoa sp.

$\begin{array}{llllllllll}0 & 0 & 0 & 3 & 0 & 2 & 0 & 0 & 0 & 0\end{array}$

$\begin{array}{llllll}0 & 0 & 0 & 0 & 0 & 14\end{array}$

\subsection{8}

$1.98 \quad 1$

$\begin{array}{ll}1.98 & 1 \\ 19.84 & 4\end{array}$

$14.66 \quad 3$

$2.78 \quad 2$

5.56

Bivalvia

Musculium lacustre

Pisidium nitidum

$\begin{array}{llllllllll}0 & 0 & 0 & 0 & 0 & 0 & 0 & 0 & 1 & 0\end{array}$

$\begin{array}{lllll}0 & 0 & 0 & 0 & 0\end{array}$

5.56

$\begin{array}{lll}0.4 & 2 & 11.11\end{array}$

$\begin{array}{lllll}0 & 0 & 0 & 48 & 0\end{array}$

$\begin{array}{lllll}0 & 0 & 0 & 0 & 0\end{array}$

$\begin{array}{lllll}0 & 0 & 0 & 0 & 0\end{array}$

$\begin{array}{llllll}0 & 0 & 0 & 0 & 0 & 1\end{array}$

$\begin{array}{llllllllll}0 & 0 & 0 & 2 & 0 & 5 & 0 & 0 & 0 & 0\end{array}$

$\begin{array}{lllll}9 & 0 & 0 & 2 & 0\end{array}$

$\begin{array}{llllll}0 & 0 & 0 & 0 & 0 & 48\end{array}$

Abra sp.

$\begin{array}{llllllllll}0 & 0 & 0 & 0 & 0 & 1 & 0 & 0 & 0 & 0\end{array}$

$\begin{array}{llllll}0 & 0 & 0 & 0 & 0\end{array}$

$\begin{array}{lllll}0 & 0 & 0 & 0 & 18\end{array}$

$19.05 \quad 1 \quad 5.56$

Unio eucirrus

$\begin{array}{llllllllll}0 & 0 & 0 & 0 & 0 & 2 & 0 & 0 & 0 & 0\end{array}$

$\begin{array}{lllllllllll}0 & 0 & 0 & 0 & 0 & 0 & 0 & 0 & 0 & 0 & 2\end{array}$

$\begin{array}{lll}7.14 & 4 & 22.22\end{array}$

$0.4 \quad 1 \quad 5.56$

$0.79 \quad 1 \quad 5.56$ 
Galba truncatula'nın yüksek pH (9.6' ya kadar) değerlerinde yaşayabildiği ve insan yerleşimlerine yakın lokalitelerde bulundukları bildirilmiştir (Glöer, Yıldırım ve Kebapçı, 2015). Kavak Çayı'nda akarsu boyunca tarım ve hayvancılık faaliyetlerinin yaygın olması ve suyun pH değerlerinin (Tablo 4) bu türün bölgedeki dağılımını teşvik ettiği söylenebilir. Aynı zamanda, molluska faunasının Kavak Çayı'nda nispeten düşük çeşitlilik sergilemesinin yukarıda belirtilen çevresel kullanımlar ile ilgili olabileceği düşünülmektedir.

Kavak Çayı'nda gerçekleştirilen bu çalışmada, teşhis edilen birçok gastropod ve bivalv türünün (Bithynia sp. B. tentaculata, V. piscinalis, B. reticulatum, E. ventrosa, Rissoa sp., G. truncatula, G. piscinarum, P. acuta, $P$. carinatus, $P$. intermixtus, $R$. labiata, Pisidium sp., Abra sp., U. eucirrus) I. istasyonda bir arada bulunduklar1 görülmektedir (Tablo 2). Ölçülen su kalite parametresi bakımından SKKY'ne göre I. ve IV sınıf yüzey suyu özelliği taşıyan bu istasyonda organik kirliliğe orta derecede toleranslı taksonların bulunması (Kerney, 1999; Cabuk, Arslan ve Yılmaz, 2005; Seddon, Kebapçı ve Van Damme, 2014), bu istasyonun mollusk taksonlarının tercih ettikleri habitatları da bulundurmasıyla açıklanabilir. Çalışmanın birinci istasyonu nehir ağzı ekosistemine yakın olduğundan, tipik bir acı su türü olan E. ventrosa (Kerney, 1999) ve B. reticulatum, Rissoa sp., Abra sp. gibi bazı denizel taksonların bu ortamda diğer tatlısu molluskları ile düşük sayıda da olsa birlikte bulunmaları olağan bir durumdur. Küresel bir istilacı olan ve Kuzey Amerika kökenli P. acuta (Dillon, Wethington, Rhett ve Smith, 2005; Semenchenko, Laenko ve Razlutskii, 2008; Odabașı, Arslan, Özdilek ve Odabaș1, 2019), bu çalışmada G. truncatula' dan sonra en sık rastlanılan ikinci tür olduğu tespit edilmiştir. Akbulut vd. (2009a)'un bulgularına göre ise P. acuta Sarıçay ve Atikhisar Barajında en bol ve sık bulunan türdür.

Tablo 4

Örnekleme İstasyonlarındaki Bazı Su Kalitesi Parametreleri ortalama değerleri, standart sapmaları $( \pm)$ ve su kalitesi sinifları

\begin{tabular}{|c|c|c|c|c|c|}
\hline Parametreler & KV1 & KV2 & KV3 & KV4 & KV5 \\
\hline pH & $7.06 \pm 1.39$ & $7.01 \pm 1.82$ & $8.47 \pm 0.26$ & $7.71 \pm 1.44$ & $8.39 \pm 0.26$ \\
\hline Kalite Sinıfi & I & I & I & I & I \\
\hline E.İ $(\mu \mathrm{S} / \mathrm{cm})$ & 111.5 & 1468 & 1198 & 3278 & 3278 \\
\hline Sicaklık & $13.39 \pm 10.62$ & $16.86 \pm 4.15$ & $15.35 \pm 9.63$ & $14.73 \pm 5.87$ & $16.95 \pm 9.65$ \\
\hline Kalite Sınıfı & I & I & I & I & I \\
\hline ÇO $(\%)$ & $87.37 \pm 41.47$ & $63.65 \pm 20.17$ & $58.65 \pm 16.19$ & $53.9 \pm 15.07$ & $51.2 \pm 14.14$ \\
\hline Kalite Sınıfi & II & III & III & III & III \\
\hline ÇO $(\mathrm{mg} / \mathrm{L})$ & $6.88 \pm 1.87$ & $8.29 \pm 1.46$ & $5.91 \pm 0.37$ & $5.37 \pm 1.29$ & $4.9 \pm 0.34$ \\
\hline Kalite SInıfi & II & I & III & III & III \\
\hline TDS (mg/L) & $306.69 \pm 407.22$ & $443.77 \pm 574.49$ & $758 \pm 118.8$ & $835 \pm 176.74$ & $555 \pm 72.13$ \\
\hline Kalite Sinıfi & I & I & II & II & II \\
\hline $\mathrm{Cl}^{-}(\mathrm{mg} / \mathrm{L})$ & 21.43 & & \multirow{2}{*}{ - } & 18.97 & \multirow{2}{*}{ - } \\
\hline Kalite Sinıfı & I & & & I & \\
\hline $\mathrm{SO}_{4}^{-}(\mathrm{mg} / \mathrm{L})$ & 43.3 & \multirow[b]{2}{*}{-} & \multirow{2}{*}{-} & 36.15 & \multirow[b]{2}{*}{ - } \\
\hline Kalite Sinıfi & I & & & I & \\
\hline $\mathrm{NO}_{3}(\mathrm{mg} / \mathrm{L})$ & 3.42 & \multirow[b]{2}{*}{-} & \multirow[b]{2}{*}{ - } & 13.21 & \multirow[b]{2}{*}{ - } \\
\hline Kalite Sinıfi & I & & & III & \\
\hline $\mathrm{Na}^{+}(\mathrm{mg} / \mathrm{L})$ & $12.7 \pm 10.03$ & $5.87 \pm 1.42$ & $14.53 \pm 15.14$ & $3.42 \pm 1.75$ & $13.42 \pm 13.59$ \\
\hline Kalite Sinıfi & I & I & I & I & I \\
\hline
\end{tabular}


Tablo 4 (Devami)

\begin{tabular}{|c|c|c|c|c|c|}
\hline Parametreler & KV1 & KV2 & KV3 & KV4 & KV5 \\
\hline$\overline{C d}(\mu \mathrm{g} / \mathrm{L})$ & 0.31 & \multirow[b]{2}{*}{-} & 0.19 & $1.37 \pm 2.75$ & \multirow{2}{*}{-} \\
\hline Kalite Sınıfi & I & & I & I & \\
\hline $\mathrm{Pb}(\mu \mathrm{g} / \mathrm{L})$ & $11.29 \pm 7.56$ & 8.6 & $6.72 \pm 5.18$ & $9.3 \pm 3.74$ & $1.53 \pm 2.16$ \\
\hline Kalite Sinıfi & II & I & I & I & I \\
\hline $\mathrm{Cu}(\mu \mathrm{g} / \mathrm{L})$ & $3.04 \pm 1.52$ & $1.95 \pm 1.43$ & $2.11 \pm 2.9$ & $1.79 \pm 1.33$ & $3.03 \pm 1.6$ \\
\hline Kalite Sınıfi & I & I & I & I & I \\
\hline $\operatorname{Cr}(\mu \mathrm{g} / \mathrm{L})$ & $0.8 \pm 0.36$ & 0.11 & 0.3 & 0.41 & 0.53 \\
\hline Kalite Sinıfi & I & I & I & I & I \\
\hline Mn $(\mu \mathrm{g} / \mathrm{L})$ & $142.68 \pm 211.08$ & $4.08 \pm 1.63$ & $9.53 \pm 5.11$ & $9.21 \pm 2.97$ & $5.91 \pm 0.007$ \\
\hline Kalite Sınıfı & III & I & I & I & I \\
\hline $\mathrm{Ba}(\mu \mathrm{g} / \mathrm{L})$ & $68.5 \pm 37.38$ & $34.26 \pm 3.82$ & $44.38 \pm 16.56$ & $64.52 \pm 33.52$ & $32.97 \pm 0.42$ \\
\hline Kalite Sinıfi & I & I & I & I & I \\
\hline $\mathrm{Al}(\mathrm{mg} / \mathrm{L})$ & $41.47 \pm 40.9$ & $51.01 \pm 52.19$ & $55.29 \pm 45.72$ & $40.75 \pm 32.47$ & $18.25 \pm 1.66$ \\
\hline Kalite Sınıfı & IV & IV & IV & IV & IV \\
\hline $\mathrm{Fe}(\mu \mathrm{g} / \mathrm{L})$ & 31.45 & 13.33 & 2.45 & 21.89 & 9.13 \\
\hline Kalite Sınıfi & I & I & I & I & I \\
\hline Co $(\mathrm{mg} / \mathrm{L})$ & $1.17 \pm 0.35$ & $0.96 \pm 0.43$ & $0.99 \pm 0.81$ & $0.6 \pm 0.41$ & $0.35 \pm 0.09$ \\
\hline Kalite Sınıfi & I & I & I & I & I \\
\hline$\overline{Z n}(\mu \mathrm{g} / \mathrm{L})$ & $6.38 \pm 8.23$ & 2.91 & 0.45 & 3.47 & \multirow{2}{*}{ ALA } \\
\hline Kalite Sınıfi & I & I & I & I & \\
\hline $\mathrm{Ni}(\mathrm{mg} / \mathrm{L})$ & $4.75 \pm 5.26$ & 4.1 & 0.62 & $0.87 \pm 1.38$ & \multirow{2}{*}{ ALA } \\
\hline Kalite Sinıfı & I & I & I & I & \\
\hline$\overline{M g}(\mathrm{mg} / \mathrm{L})$ & & \multirow[b]{2}{*}{-} & \multirow{2}{*}{-} & $2.60 \pm 1.49$ & $2.68 \pm 0.12$ \\
\hline Kalite Sınıfı & & & & I & I \\
\hline$B(\mathrm{mg} / \mathrm{L})$ & & & & $79.28 \pm 33.17$ & $35.97 \pm 12.33$ \\
\hline Kalite Sinıfi & & & & I & I \\
\hline $\mathrm{Ca}(\mathrm{mg} / \mathrm{L})$ & $1.17 \pm 0.18$ & $0.96 \pm 0.23$ & $0.99 \pm 0.46$ & $0.6 \pm 0.03$ & $0.35 \pm 0.11$ \\
\hline Kalite Sinıfı & I & I & I & I & I \\
\hline $\mathrm{K}(\mathrm{mg} / \mathrm{L})$ & $8.11 \pm 2.24$ & $5.6 \pm 0.9$ & $5.52 \pm 3.42$ & 2.11 & 2.77 \\
\hline Kalite Sımıfi & I & I & I & I & I \\
\hline
\end{tabular}

ALA: Analiz Limiti Altında

Elde edilen verilere göre, Kavak Çayı'ndaki birey sayısı bakımından en bol bulunan bivalv türü Pisidium nitidum'dur. Kuiper vd. (1989)'a göre Kuzey Avrupa'da daha yaygın olan P. nitidum, soğuk sulara toleranslı bir türdür. (Akbulut vd., 2009a) tarafından Çanakkale ili iç sularından varlığı bildirilen bu türün, Türkiye'deki diğer havzalardan varlığı bilinmemektedir.

Kavak çayında belirlenen örnekleme istasyonlarındaki taksonların tolerans değerlerinin Hilsenhoff ölçeğine göre (Mandaville, 2002) genelde yüksek toleranslı ve toleranslı kategorisinde yer aldıkları görülmektedir (Tablo 5). Bu bakımdan bu çalışmadaki örnekleme istasyonlarının organik kirleticilerin baskısı altında olduğu söylenebilir. Yerinde ve laboratuvarda ölçülen su kalitesi parametrelerinden ÇO yoğunluk ve yüzde doygunluğu, $\mathrm{NO}_{3}$ ve $\mathrm{Al}$ değerlerinin SKKY'ne göre çoğunlukla III. ve IV. sınıf su kalitesi özelliği sergilemiştir (Tablo 4). Bu durumun bölgedeki tarımsal faaliyetlerin yoğunluğundan ve yerleşim yerlerindeki alt yapı 
eksikliğinden (Anonim, 2013: 35) kaynaklandığ1 öngörülmektedir. Alüminyum elementinin sudaki birincil kaynağı toprak ve kayaçlardır (Miller, Kopfler, Kelty, Stober ve Ulmer, 1984), bu bağlamda, Kavak Çayının üst havzasında deva eden baraj inşaatının (Çokal Barajı) alüminyum değerlerini yükselttiği düşünülebilir.

Tablo 5

Teşhis edilen Molluska taksonlara ait Hilsenhoff biotik indeksine göre taksa tolerans değerleri

\begin{tabular}{|c|c|c|c|c|c|c|c|c|}
\hline Taksonlar & $\begin{array}{c}\text { Tolerans } \\
\text { Değeri }\end{array}$ & $\begin{array}{c}\text { İndikatör/ } \\
\text { Su kalitesi* }\end{array}$ & Taksonlar & $\begin{array}{c}\text { Tolerans } \\
\text { Değeri }\end{array}$ & $\begin{array}{c}\text { Indikatör/ } \\
\text { Su kalitesi* }\end{array}$ & Taksonlar & $\begin{array}{c}\text { Tolerans } \\
\text { Değeri }\end{array}$ & $\begin{array}{c}\text { İndikatör/ } \\
\text { Su kalitesi* }\end{array}$ \\
\hline $\begin{array}{l}\text { G. } \\
\text { truncatula }\end{array}$ & 6 & $\mathrm{~T} / \mathrm{O}$ & $\begin{array}{l}\text { B. } \\
\text { tentaculata }\end{array}$ & 8 & $\mathrm{YT} / \mathrm{S}$ & M. lacustre & 6 & $\mathrm{~T} / \mathrm{O}$ \\
\hline $\begin{array}{l}\text { G. } \\
\text { piscinarum }\end{array}$ & 8 & YT/S & Bithynia sp. & 8 & $\mathrm{YT} / \mathrm{S}$ & P. nitidum & 8 & $\mathrm{YT} / \mathrm{S}$ \\
\hline P. acuta & 8 & $\mathrm{YT} / \mathrm{S}$ & $\begin{array}{l}V . \\
\text { piscinalis }\end{array}$ & 8 & $\mathrm{YT} / \mathrm{S}$ & Pisidium sp. & 6 & $\mathrm{~T} / \mathrm{O}$ \\
\hline $\begin{array}{l}P . \\
\text { carinatus }\end{array}$ & 6 & $\mathrm{~T} / \mathrm{O}$ & $\begin{array}{l}B . \\
\text { reticulatum }\end{array}$ & yok & & Abra sp. & yok & \\
\hline $\begin{array}{l}P . \\
\text { intermixtus }\end{array}$ & 6 & $\mathrm{~T} / \mathrm{O}$ & $\begin{array}{l}\text { E. } \\
\text { ventrosa }\end{array}$ & yok & & U. eиcirrus & 6 & $\mathrm{~T} / \mathrm{O}$ \\
\hline $\begin{array}{l}R . \\
\text { labiata }\end{array}$ & 6 & $\mathrm{~T} / \mathrm{O}$ & Rissoa sp. & yok & & & & \\
\hline
\end{tabular}

*: T (toleransl1), YT (yüksek toleransl1), O (orta; organik kirlilik mevcut), S (sağlıksız; belirgin organik kirlilik mevcut) 


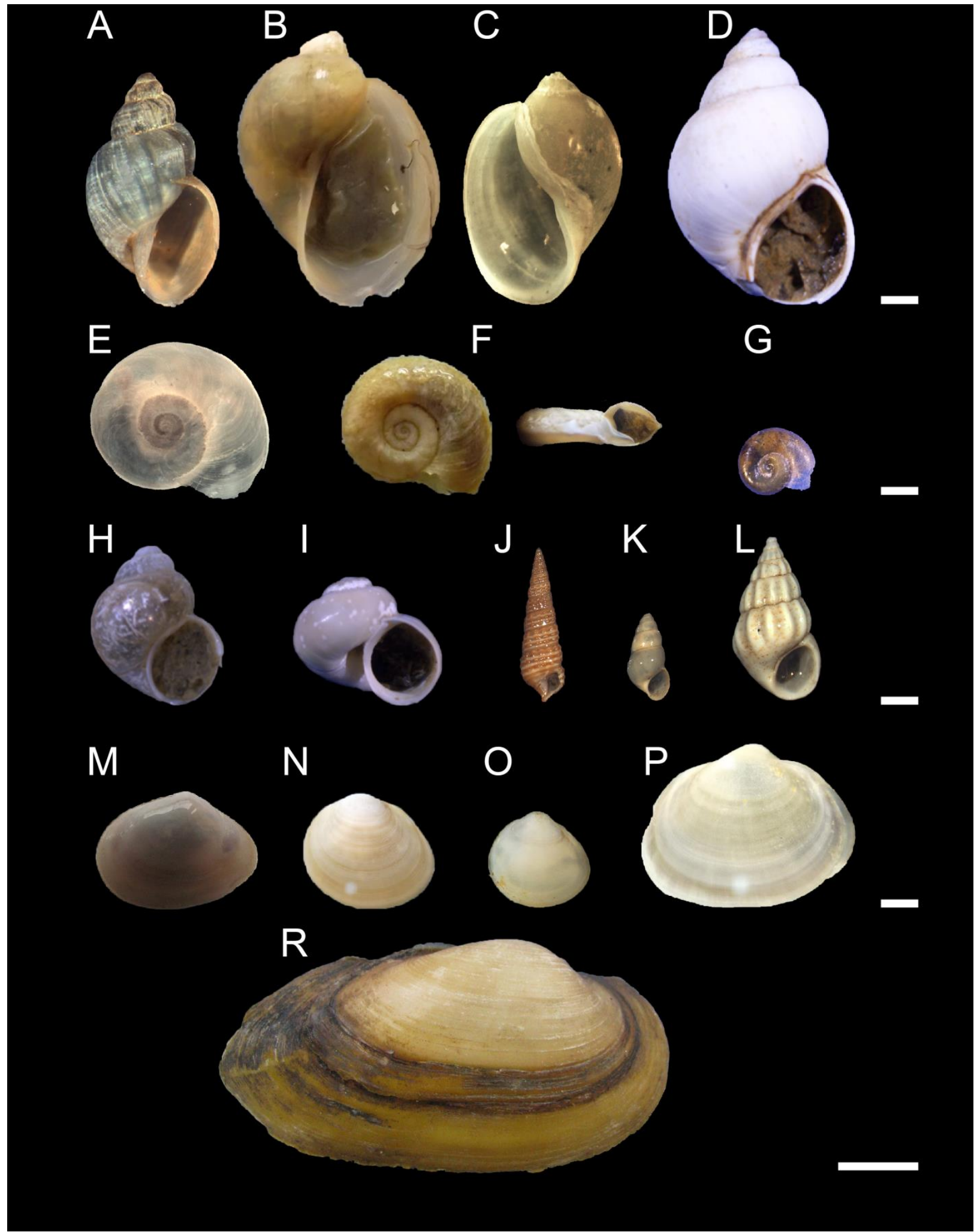

Şekil 2. Çalışma alanından teşhis edilen taksonlar. A: Galba truncatula, B: Radix labiata, C: Physella acuta, D: Bithynia tentaculata, E: Planorbis intermixtus, F: Planorbis carinatus, G: Gyraulus piscinarum, H: Bithynia sp., I: Valvata piscinalis, J: Bittium reticulatum, K: Ecrobia ventrosa, L: Rissoa sp., M: Abra sp., N: Pisidium nitidum, O: Pisidium sp., P: Musculium lacustre, R: Unio eucirrus (Ölçek Barı A-P: $1 \mathrm{~mm}, \mathrm{R}: 1 \mathrm{~cm}$ ) 


\section{Sonuçlar}

Coğrafi olarak Trakya bölgesinde yer alan ve Marmara Havzasının bir akarsu ekosistemi olan Kavak Çayı'nda gerçekleştirilen bu çalışmada teşhis edilen mollusk faunası taksonları çalışma bölgesinde ilk kez bildirilmiştir. Teşhis edilen taksonların genel olarak toleranslı ve yüksek toleranslı oldukları belirlenmiştir. Buna rağmen istilacı özellik sergileyen mollusk taksonlarının bulunmayışı, ya da düşük birey sayısı ile temsil edilmesi (P. acuta gibi) önemli bir dağılım ve popülasyon yapısı bulgusu olarak değerlendirilmiştir. Çevresel arazi kullanımı bakımından, Kavak Çayı tarım alanlarının ve bazı yerleşim alanlarının etkisi altındadır. Bu durum, bazı su kalitesi verilerine yansıdığı gibi, ağırlıklı olarak ekolojik toleransı yüksek molluskların varlığı ile de kendini göstermiştir. Tatlısu habitatlarının sucul ekosistem içerisinde en fazla baskı altındaki habitatlar oldukları belirtilmektedir (Angelier, 2002; Mason, 2002). Çalışma alanımızda elde edilen veriler dikkate alındığında, Kavak Çayı'nda nitrat, alüminyum ve çözünmüş oksijen gibi bazı su kalitesi parametrelerinin su kalite sınıfını düşürdüğ̈̈ ve tespit edilen mollusk taksonlarının genelde toleranslı özellikte olduğu görülmektedir. Gerekli tedbirlerin alınmasıyla su kalitesinde ve sucul organizma komünitesinde olumlu gelişmeler kaydedilmesi öngörülmektedir.

\section{Teşekkür}

Bu Yüksek Lisans Tezi, Çanakkale Onsekiz Mart Üniversitesi, BAP birimince FYL-2015-676 kodu ile finanse edilmiştir. Ayrıca bu çalışmanın son haline gelmesinde katkılarını sunan hakemlere, özellikle Unio eucirrus'un teşhisinde yardımcı olan hakeme çok teşekkür ederiz.

\section{Yazar Katkıları}

Demet Bal: Saha örneklemesi, Laboratuvar uygulama ve analizleri konularında katkı sağlamıştır.

Deniz Anıl Odabaşı: Projenin yürütülmesi, Metodoloji seçimi ve uygulanması ve Taslak metnin yazımı konularında katkı sağlamıştır.

Aytuğ Zilifli: Çalışmanın istatistiksel analizlerini yapmış, taslak metin yazımında katkı sağlamıştır.

\section{Çıkar Çatışması}

Yazarlar çıkar çatışması beyan etmemektedir.

\section{Kaynaklar}

Akbulut, M., Çelik, E. Ş., Odabaşı, D. A., Kaya, H., Selvi, K., Arslan, N. ve Sağır-Odabaşı, S. (2009b). Seasonal distribution and composition of benthic macroinvertebrate communities in Menderes Creek, Çanakkale, Turkey. Fresenius Environmental Bulletin, 18(11a), 2136-2145. Erişim adresi: https://www.researchgate.net/profile/Kahraman-

Selvi/publication/259384032 Seasonal distribution and composition of benthic macroinvertebrate communities_in_menderes_creek_Canakkale_TURKEY/links/0c96052ba1e9bbec4a000000/Seasonaldistribution-and-composition-of-benthic-macroinvertebrate-communities-in-menderes-creekCanakkale-TURKEY.pdf

Akbulut, M., Odabaşı, D. A., Kaya, H., Çelik, E. Ş., Yıldırım, M. Z., Odabaşı, S. S. ve Selvi, K. (2009a). Changing of Molusca Fauna In Comparison With Water Quality: Sarıçay Creek and Atikhisar Reservoir Models (Çanakkale-Turkey), Journal of Animal and Veterinary Advances 8(12): 2699-2707. Erişim adresi: https://www.researchgate.net/publication/259384114

Akbulut, M., Odabaşı, S. S., Odabaşı, D. A. ve Çelik, E. Ş. (2006). Çanakkale İli'nin Önemli İçsuları ve Kirletici Kaynakları. Su Ürünleri Dergisi, 23(1), 9-15. Erişim adresi: http://www.egejfas.org/en/download/article-file/57667

Angelier, E. (2002). Ecologia de las aguas corrientes. Madrid: Acribia. Erişim adresi: https://www.editorialacribia.com/libro/ecologia-de-las-aguas-corrientes_54170/

Anonim. (2004). Su Kirliliği Kontrolü Yönetmeliği. Resmi Gazete Tarihi 31.12.2004. Resmi Gazete 
Say1s1.25687. Erişim adresi: http://www.dogacevre.com.tr/files/pdf/81.pdf

Anonim. (2013). Çanakkale Il Çevre Durumu Rapor 2012: Çanakkale İl Çevre ve Şehircilik Md.. Erişim Adresi: https://webdosya.csb.gov.tr/db/ced/editordosya/Canakkale icdr2013.pdf

Bilgin, F. H. (1980). Batı Anadolu'nun Bazı Önemli Tatlı Sularından Toplanan Mollusca Türlerinin Sitematiği ve Dağılışı. Diyarbakır Üniversitesi Tip Fakültesi Dergisi, 8(2): 1-64.

Borcherding, J. ve Volpers, M. (1994). The "Dreissena-Monitor" first results on the application of the biological early warning system in the continuous monitoring of water quality. Water Science and Technology, 29(3), 199-201. https://doi.org/10.2166/wst.1994.0099

Bulut, M. ve Birben, Ü. (2019). AB Su Çerçeve Direktifinin Türkiye'de su kaynakları yönetimine etkisi. Türkiye Ormancılık Dergisi, 20(3), 221-233. https://doi.org/10.18182/tjf.562550

Çabuk, Y., Arslan, N. ve Y1lmaz, V. (2005). Species Composition and Seasonal Variations of the Gastropoda in Upper Sakarya River System (Turkey) in Relation to Water Quality. Acta Hydrochimica et Hydrobiologica, 6(32), 393-400. https://doi.org/10.1002/aheh.200300544

Demirbaş, A. (2001). Biomass resource facilities and biomass conversion processing for fuels and chemicals. Energy Conversion and Management, 42(11), 1357-1378. https://doi.org/10.1016/S0196$\underline{8904(00) 00137-0}$

Dillon, R. T., Wethington, A. M., Rhett, J. M. ve Smith, T. P. (2005). Populations of the European freshwater pulmonate Physella acuta are not reproductively isolated from America Physella heterostropha or Physella integra. Invertebrate Biology, 3(121), 226-234. https://doi.org/10.1111/j.17447410.2002.tb00062.x

Glöer, P. (2015). Süßwassermollusken-Ein Bestimmungsschlüssel für die Muscheln und Schnecken im Süßwasser der Bundesrepublik Deutchland. Göttingen, Deutscher Jugendbund für Naturbeobachtung.

Glöer, P., Yıldırım, M. Z. ve Kebapçı, Ü. (2015). Description of two new species of Pseudamnicola from southern Turkey ( Mollusca: Gastropoda: Hydrobiidae). Zoology in the Middle East, 2(61), 1-5. https://doi.org/10.1080/09397140.2015.1008189

Goldberg, E. G. (1986). The mussel watch concept. Environmental Monitoring and Assessment, 7, 91-103. https://doi.org/10.1007/BF00398031

Gözler, A. M. ve Baytaşoğlu, H. (2020). Mollusca Fauna of the Çoruh River and Its Tributaries. Journal of Anatolian Environmental And Animal Sciences, 5(2), 185-190. https://doi.org/10.1080/09397140.2015.1008189

Gürlek, M. E. (2017). Three new truncatelloidean gastropod species from Turkey (Caenogastropoda: Littorinimorpha). Turkish Journal of Zoology, 41(6), 991-997. https://doi.org/10.3906/zoo-1701-37

Gürlek, M. E., Koşal- Şahin, S., Dökümcü, N. ve Yıldırım, M. Z. (2019). Checklist of the freshwater mollusca of Turkey (Mollusca: Gastropoda, Bivalvia). Fresenius Enviromental Bulletin, 28(4), 2992-3013. Erişim adresi: https://www.researchgate.net/profile/MustafaGuerlek/publication/332422818_CHECKLIST_OF_THE_FRESHWATER_MOLLUSCA_OF_TURK EY_MOLLUSCA_GASTROPODA_BIVALVIA/links/5cb482c192851c8d22eca5a0/CHECKLISTOF-THE-FRESHWATER-MOLLUSCA-OF-TURKEY-MOLLUSCA-GASTROPODABIVALVIA.pdf

Hering, D., Johnson, R. K., Kramm, S., Schmutz, S., Szoszkiewicz, K. ve Verdonschot, P. F. M. (2006). Assessment of European streams with diatoms, macrophytes, macroinvertebrates and fish: a comparative metric-based analysis of organism response to stress. Freshwater Biology 51(9), 17571785. https://doi.org/10.1111/j.1365-2427.2006.01610.x

Karr, J. R. (1999). Defining and measuring river health. Freshwater Biology, 41(2), 221-234. Erişim adresi: https://doi.org/10.1046/j.1365-2427.1999.00427.x

Karslığlu, E., Baba A. ve Deniz, O. (2004). Environmental Problems of Çanakkale Province. Proceeding of 5th National Ecology and Environment Congress (pp 513-538). Bolu, Turkey.

Kazancı, N., Türkmen, G., Ertunç, Ö., Ekingen, P., Öz, B. ve Gültutan, Y. (2010). Assessment of ecological quality of Yeşilırmak River (Turkey) by using macroinvertebrate-based methods in the content of Water Framework Directive. Review of Hydrobiology, 3(2), 89-110. Erişim adresi: https://www.researchgate.net/publication/235933586

Kebapçı, Ü. ve Yıldırım, M. Z. (2010). Freshwater snails fauna of the lakes region (Göller Bölgesi), Turkey. Oltenia Studii şi comunicări. Ştiinţele Naturii, 26(2): 75-83. Erişim adresi: http://biozoojournals.ro/oscsn/cont/26_2/IZ02.Kebaci.pdf

Kelkit, A. ve Öztürk, C. (2005) Kavak Deltası (Çanakkale)'nın Korunan Alanlar Açısından İncelenmesi. Korunan Doğal Alanlar Sempozyumu (pp. 233-236). Isparta, Türkiye. Erişim adresi: 
http://ormanweb.isparta.edu.tr/kdas/belgeler/posterbildiriler/56.pdf

Kenney, M. A., Sutton-Grier, A. E., Smith, R. F. ve Gresens, S. E. (2009). Benthic macroinvertebrates as indicators of water quality: The intersection of science and policy. Terrestrial Arthropod Reviews, 2(2), 99. https://doi.org/10.1163/187498209X12525675906077

Kerney, M. (1999). Atlas of the land and Frestwater molluscs of Britain and Ireland. Colchester, England: Harley Books. Erişim Adresi: https://brill.com/view/title/23971

Kılıçaslan, I. ve Özbek, M. (2010). Contributions to the knowledge on the distribution of freshwater Mollusca species of Turkey. Review of Hydrobiology, 3(2), 127-144. Erişim Adresi: https://www.researchgate.net/profile/Murat-

Ozbek/publication/257941767_Contributions_to_the_knowledge_on_the_distribution_of_freshwater_ Mollusca_species_of_Turkey/links/00b7d526669a9e1735000000/Contributions-to-the-knowledge-onthe-distribution-of-freshwater-Mollusca-species-of-Turkey.pdf

Killeen, I. J., Aldridge, D., Oliver, G. ve Council, F. S. (2004). Freshwater bivalves of Britain and Ireland. Field Studies Council. UK.

Korniushin, A. V. (1996). Bivalve molluscs of the superfamily Pisidioidea in the Palaearctic region: fauna, systematics, phylogeny. National Academy of Science of Ukraine; Schmalhausen Institute of Zoology.

Koşal-Şahin, S. ve Zeybek, M. (2016). Distribution of Mollusca fauna in the streams of Tunceli Province (East Anatolia, Turkey) and its relationship with some physicochemical parameters. Turkish Journal of Fisheries and Aquatic Sciences, 16: 187-195. http://doi.org/10.4194/1303-2712-v16 119

Koşal-Şahin, S., Bahadır-Koca, S. ve Yıldırım, M. Z. (2012). New Genera Anatolidamnicola and Sivasi (Gastropoda: Hydrobiidae) from Sivas and Malatya (Turkey). Acta Zoologica Bulgarica, 64(4), 341346. Erișim Adresi: https://www.researchgate.net/profile/Serap-KosalSahin/publication/289105219 New Genera Anatolidamnicola and Sivasi Gastropoda Hydrobiidae from_Sivas_and_Malatya_Turkey/links/56ebde6a08aefd0fc1c7171b/New-Genera-Anatolidamnicolaand-Sivasi-Gastropoda-Hydrobiidae-from-Sivas-and-Malatya-Turkey.pdf

Kuiper, J. J., Økland, K. A., Knudsen, J., Koli, L., Proschwitz, T. V. ve Valovirta, I. (1989). Geographical distribution of the small mussels (Sphaeriidae) in North Europe (Denmark, Faroes, Finland, Iceland, Norway and Sweden). Annales Zoologici Fennici, 73-101. Erişim Adresi: https://www.jstor.org/stable/23736060?casa_token=-

X5kTCw8Ji8AAAAA\%3At7tIJtQ pnLR3GZiqvbws7I5SUyOsF1Q7a4nGi9G6i8vB63GKIn jIW94S ZrxxRWHned56rrusgKSDP-

DhkA0Of1nJnvggS5YmIJBgkHIcA9pY7FXKJl\&seq=1\#metadata info tab contents

Lopes-Lima, M., Gürlek, M. E., Kebapçı, Ü., Şereflişan, H., Yanık, T., Mirzajani, A., Neubert, E., Prié, V., Teixeira, A., Gomes-dos-Santos, A., Barros-García, D., Bolotov, I.N., Kondakov, A. V., Vikhrev, I. V., Tomilova, A. A., Özcan, T., Altun, A., Gonçalves, D. V., Bogan, A. E. ve Froufe, E. (2021). Diversity, biogeography, evolutionary relationships, and conservation of Eastern Mediterranean freshwater mussels (Bivalvia: Unionidae). Molecular Phylogenetics and Evolution, 163, 107261. https://doi.org/10.1016/j.ympev.2021.107261

Mandaville, S. M. (2002). Benthic Macroinvertebrates in Freshwaters Taxa Tolerance Values, Metrics and Protocols. Nova Scotia: Soil ve Water Conservation Society of Metro Halifax, Canada. Erişim Adresi: http://citeseerx.ist.psu.edu/viewdoc/download?doi=10.1.1.516.2776\&rep=rep1\&type=pdf

Mason, C. F. (2002). Biology of Freshwater Pollution. Newyork: Pretince-Hall (Pearson Education). ISBN: 0130906395,9780130906397

Miller, R. G., Kopfler, F. C., Kelty, K. C., Stober, J. A. ve Ulmer, N. S. (1984). The occurrence of aluminum in drinking water. Journal-American Water Works Association, 76(1), 84-91. https://doi.org/10.1002/j.1551-8833.1984.tb05267.x

Odabaşı, D. A., Kebapçı, Ü. ve Akbulut, M. (2013). Description of a new Pseudobithynia n. sp.(Gastropoda: Bithyniidae) from Northwest Turkey. Journal of Conchology, 41(4), 527-532. https://www.researchgate.net/profile/Deniz-

Odabasi/publication/259578973 Description of a new pseudobithynia $\mathrm{n}$ sr Gastropoda Bithyniida e_from_Northwest_Turkey/links/0c96052cbb4c55cfd6000000/Description-of-a-new-pseudobithynian-sr-Gastropoda-Bithyniidae-from-Northwest-Turkey.pdf

Odabaş1, D. A. ve Georgiev, D. (2014). Bythinella kazdaghensis sp. n.(Gastropoda: Rissooidea) from the Mount Ida (Kaz Dağı)-Northwestern Turkey. Acta Zooogica Bulgarica, 66(1), 21-24. Erişim adresi: https://www.researchgate.net/profile/Dilian-

Georgiev/publication/261251591_Bythinella_kazdaghensis_sp_n_Gastropoda_Rissooidea_from_the_ 
Mount_Ida_Kaz_Dagi_Northwestern_Turkey/links/00463533b2fab81b1b000000/Bythinellakazdaghensis-sp-n-Gastropoda-Rissooidea-from-the-Mount-Ida-Kaz-Dagi-Northwestern-Turkey.pdf

Odabaş1, D. A., Akay, E., ve Koyuncuoğlu, S. (2020). Pseudamnicola thalesi sp. n.(Gastropoda: Truncatelloidea: Hydrobiidae), a new freshwater gastropod species from Western Turkey. Zoology in the Middle East, 66(2), 140-144. https://doi.org/10.1080/09397140.2020.1739370

Odabaş1, S. ve Büyükateş, Y. (2009). Klorofil-a, çevresel parametreler ve besin elementlerinin günlük değişimleri: Sarıçay akarsuyu örneği (Çanakkale, Türkiye). Ekoloji, 19(73), 76-85. https://doi.org/10.5053/ekoloji.2009.7310

Odabaşı, S., Arslan, N., Özdilek, Ş. Y. ve Odabaşı, D. A. (2019). An Invasion Report of The New Zealand Mud Snail, Potamopyrgus antipodarum (Gray, 1843) in Turkish Freshwaters: Delice River and Kocabaş Stream. Journal of Limnology and Freshwater Fisheries Research, 5(3) 213-219. https://www.doi.org/10.17216/Limnofish.486626

Öktener, A. (2004). Sinop ve Bafra'da Bazı Tatlısulardaki Mollusca Türleri Üzerine Ön Araştırma. Gazi Üniversitesi Journal of Science, 17(2), 21-30.

Öztürk, B., Önen, M. ve Doğan, A. (2008). Türkiye Denizel Mollusca Türleri Tayin Atlası. Ankara, The Scientific and Technical Research Council of Turkey (TÜBITAK) 103T154, 468.

Rosenberg, D. M. (1998). A national aquatic ecosystem health program for Canada: We should go against the flow. Bulletin of Entomological Society Canada, 30(4), 144-152.

Rosenberg, D. M. ve Resh, V. H. (1993). Introduction to freshwater biomonitoring and benthic macroinvertebrates, New York Chapman and Hall. https://doi.org/10.2307/2404174

Roth, J. R. (1839). Molluscorum species, quas in itinere per Orientem facto comites Schuberti M. Erdl et JR Roth collegerunt. Diss. inaug. Wolf.

Salanki, J., Salanki A. F. Kamardina T. ve Rozsa K. S. (2003). Molluscs in biological monitoring of water quality, Toxicology Letters 140-141, 403-410. https://doi.org/10.1016/S0378-4274(03)00036-5

Salanki, J. (1989). New avenues in the biological indication of environmental pollution. Acta Biologica Academiae Scientiarum Hungaricae 40, 295-328. Erişim adresi: https://europepmc.org/article/med/2486724

Sands, A. F., Glöer, P., Gürlek, M. E., Albrecht, C. ve Neubauer, T. A. (2020) A revision of the extant species of Theodoxus (Gastropoda, Neritidae) in Asia, with the description of three new species. Zoosystematics and Evolution, 96 (1), 25-66. https://doi.org/10.3897/zse.96.48312

Sarı, H. M., Balık, S., Ustaoğlu, M. R. ve Ilhan, A. (2006). Distribution and ecology of freshwater ichthyofauna of the Biga Peninsula, North-western Anatolia, Turkey. Turkish Journal Zoology 30, 35-45. Erişim adresi: https://journals.tubitak.gov.tr/zoology/issues/zoo-06-30-1/zoo-30-1-5-0411-4.pdf

Schütt, H. (1965). Zur Systematik und ökologie Türkischer Süsswasserprosobranchier. Zoologische mededelingen, 41(3) 43-73. Erişim adresi: https://repository.naturalis.nl/pub/318817

Schütt, H. ve Bilgin, F. H. (1970). Pseudamnicola geldiayana n. sp., a spring-inhabiting snail of the Anatolian Plateau. Archiv für Molluskenkunde, 100, 151-158.

Schütt, H. (1964). Die Molluskenfauna eines reliktiiren Quellsees der südlichen Türkei, Archive für Molluskenkünde, 93:173-180.

Schütt, H. ve Şeşen. R. (1993). Pseudamnicola species and other Freshwater Gastropods (MolluscaGastropoda) from East Anatolia (Turkey), the Ukraine and Lebanon, Basteria, 57:161-171. Erişim adresi: https://natuurtijdschriften.nl/pub/597025

Seddon, M. B., Kebapçı, Ü. ve Van Damme, D. (2014). Valvata piscinalis. The IUCN Red List of Threatened Species. https://dx.doi.org/10.2305/IUCN.UK.2014-1.RLTS.T156186A42435636.en.

Semenchenko, V., Laenko, T. ve Razlutskii, V. (2008). A New Record of the North American gastropod Physella acuta (Draparnaud, 1805) from the Neman Stream Basin, Belarus. Aquatic Invasions, 3(3), 359-360. https://doi.org/10.3391/ai.2008.3.3.14

Stanczykowska, A., Lewandowski, K. ve Ejsmont-Karabin J. (1983). Biotic structure and processes in the lake system of R. Jorka watershed (Masurian Lakeland, Poland). IX. Occurrence and distribution of molluscs with special consideration to Dreissena polymorpha (Pall.). Ekologia Polska, 31(3) 761-780. Erişim adresi: https://pascal-francis.inist.fr/vibad/index.php?action=getRecordDetail\&idt=9571500

Sturany, R. (1894). Zur Molluskenfauna der europaischen Türkei, Ann. des K. K. Naturhist. Hofmuseums, Separatabdruck aus Band 9(3-4): 369-394. Erişim adresi: https://www.jstor.org/stable/41767771

Tomilova, A. A., Lyubas, A. A., Kondakov, A. V., Konopleva, E. S., Vikhrev, I. V., Gofarov, M. Y., ... ve Bolotov, I. N. (2020). An endemic freshwater mussel species from the Orontes River basin in Turkey and Syria represents duck mussel's intraspecific lineage: Implications for conservation. Limnologica, 
84, 125811. https://doi.org/10.1016/j.limno.2020.125811

Ustaoğlu, M. R., Balık, S. ve Özbek, M. (2001). Işı1klı Gölü (Çivril-Denizli)'nün Mollusca faunası. Ege Journal of Fisheries and Aquatic Sciences, 18(1-2), 135-139. Erişim adresi: http://www.egejfas.org/en/download/article-file/58099

Ustaoğlu, M. R., Balık, S. ve Özbek, M. (2003). Yuvarlakçay'ın (Köyceğiz-Muğla) Mollusca Faunası. E.Ü. Su Ürünleri Dergisi, 20(3): 433-438. Erişim adresi: http://www.egejfas.org/en/download/articlefile/57992

Yıldırım M.Z. (1999). Türkiye Prosobranchia (Gastropoda: Mollusca) türleri ve zoocoğrafik yayılışları 1. Tatlı ve Ac1 Sular. Turkish Journal of Zoology, 23(3), 877-900. Erişim adresi: https://dergipark.org.tr/tr/download/article-file/135345

Yıldırım, M. Z., Kebapçı, Ü., Koca, S. B. ve Yüce, A. (2015). New Bythinella (Gastropoda, Bythinellidae) species from western Turkey. ZooKeys, 481, 1-13. https://doi.org/10.3897/zookeys.481.8225

Yıldırım, M. Z., Gümüş, B. A., Kebapçı, Ü. ve Koca, S. B. (2006). The basommatophoran pulmonate species (Mollusca: Gastropoda) of Turkey. Turkish Journal of Zoology, 30(4), 445-458. Erişim adresi: https://journals.tubitak.gov.tr/zoology/issues/zoo-06-30-4/zoo-30-4-14-0512-8.pdf 